BULLETIN OF THE

AMERICAN MATHEMATICAL SOCIETY

Volume 77, Number 6, November 1971

\title{
SOME NEW RESULTS IN THE KOLMOGOROV-SINAI THEORY OF ENTROPY AND ERGODIC THEORY ${ }^{1}$
}

\author{
BY DONALD S. ORNSTEIN
}

Introduction. Origins of ergodic theory. Our results will be concerned with 1-1 invertible transformations, $T$, of a measure space $X$, of total measure 1 , where $T$ and $T^{-1}$ transform each measurable set onto a measurable set of the same measure.

We will be concerned with the behavior of $T$ only up to sets of measure 0 . That is, we will say that $T_{1}$ acting on $X_{1}$ is isomorphic to $T_{2}$ acting on $X_{2}$ if we can find $\bar{X}_{1} \subset X_{1}$ and $\bar{X}_{2} \subset X_{2}$ of measure 1 and invariant under $T_{1}$ and $T_{2}$, respectively, and if there is an invertible, measure-preserving transformation $\psi$ of $\bar{X}_{1}$ on to $\bar{X}_{2}$ such that for all $x$ in $\bar{X}_{1}$ we have $\psi T_{1}(x)=T_{2} \psi(x)$.

Measure-preserving transformations arise in many different contexts, and I will now describe three of the more important ones.

(1) Automorphisms of compact groups. Any continuous automorphism of a compact group is a measure-preserving transformation with respect to Haar measure (see Halmos, Lectures in ergodic theory).

(2) Random processes. A stationary process can be thought of as a box that prints out one letter for each unit of time where the probability of a given letter being printed out may depend on the letters printed out in the past but is independent of the time (that is, the mechanism in the box does not change).

EXAmple 1. The box contains a roulette wheel. We spin the wheel once each unit of time and print out the result.

ExAmple 2. The box contains a roulette wheel. We look at all possibilities for three consecutive spins and divide these into two classes. Now each time we spin the wheel we look at the last three spins and print out a 1 if they fall in the first class and a 2 if they fall in the second class.

EXAMPLE 3. A teleprinter. This prints out letters where the probability of a given letter depends on what has already been printed (many possibilities will have probability 0 because they will not make sense).

An Invited Address originally presented under the title The isomorphism problem in ergodic theory at the 680th meeting of the Society in Pasadena, California, November 21, 1970; received by the editors May 21, 1971.

AMS 1970 subject classifications. Primary 28A65; Secondary 54H20.

1 This research was supported in part by NSF grant GP 21509. 
The mathematical model for a process is a transformation $T$ acting on $X$ and a partition $P$ of $X$. The reason for this is the following: We know all about the process if we know the probability of its printing out any given finite sequence. Now for each point $x \in X$ corresponds a sequence whose $i$ th term is the atom of $P$ containing $T^{i} x$. If we think of the measure on $X$ as a probability, then $P, T$ gives us the probability of any finite sequence. Furthermore, the probabilities of finite sequences determine the pair $P, T$ since the measure of the atoms of $\mathrm{V}_{-n}^{n} T^{i} P$ is determined.

The Birkhoff ergodic theorem implies that if we look at a process long enough each fixed string of letters will occur with a fixed frequency. If $T$ is ergodic (the only invariant sets have measure 0 or 1 ), this frequency will be the probability of the string. (We will only consider ergodic processes. Any other process will be some sort of average of ergodic ones.)

(3) Mechanical systems. We can represent the state of a mechanical system as a point in a high-dimensional Euclidean space (the phase space). For example, if our system is a gas confined in a box, the state of the gas can be described by giving the $x, y, z$-components of the position and momentum of each molecule. If there are $n$ molecules, the dimension of the phase space is $6 n$. If we let one unit of time elapse, each point in phase space will move to another point. This gives us a transformation of the phase space and there is a theorem of Liouville that asserts that this transformation preserves Lebesgue measure.

Studying exactly what happens to a mechanical system is in general too complicated, and various mathematicians, including Birkhoff and von Neumann, in an attempt to make the problem more treatable by ignoring motions that occurred with probability 0 , were led to studying the measure-preserving transformations associated with a mechanical system. One of the aims of ergodic theory is to determine (up to isomorphism) the measure-preserving transformations associated with specific mechanical systems.

Abstract ergodic theory : Bernoulli shifts. There is a class of transformations that play a central role in ergodic theory, namely the Bernoulli shifts. The reason for this is partly because they are in a certain sense the simplest examples of measure-preserving transformations and partly because of their role in applications.

Definition of Bernoulli shifts. If we are given positive numbers $p_{1}, \cdots, p_{k}$, where $\sum_{i=1}^{k} p_{i}=1$, we define the Bernoulli shift $\left(p_{1}, \cdots, p_{k}\right)$ as follows: Let $Y$ be a set with $k$ elements and let us give 
the $i$ th element measure $p_{i}$. Let $Y_{i},-\infty<i<+\infty$, be copies of $Y$ and let $X$ be the product of the $Y_{i}$ with the product measure. Thus each point in $X$ is a doubly infinite sequence of points in $Y$. T will act by shifting each of the above sequences. That is, $T$ will take the sequence $\left\{y_{i}^{\prime}\right\}$ into the sequence $\left\{y_{i}^{\prime}\right\}$ where $y_{i}^{\prime}=y_{i+1}$.

There is a theorem that says that any invertible, ergodic, measurepreserving transformation can be obtained if we modify the above construction by taking $Y$ to be countable and by taking some other measure invariant under the shift, instead of the product measure. Thus Bernoulli shifts are simplest in the sense that the product measure is the simplest measure invariant under the shift.

Let $P$ be the partition of $X$ into $k$ sets which is obtained as follows: $\left\{y_{i}\right\}$ and $\left\{y_{i}^{\prime}\right\}$ will be in the same atoms of $P$ if $y_{0}=y_{0}^{\prime}$. It is easy to see that $P, T$ corresponds to the process of spinning a roulette wheel with $k$ slots of widths $p_{i}$. Thus from the probability point of view Bernoulli shifts correspond to independent processes.

There is a more geometric way of describing Bernoulli shifts, which goes as follows: We will start by describing the Bernoulli shift $\left(\frac{1}{2}, \frac{1}{2}\right)$. Let $X$ be the unit square. $T$ will send the point $(x, y)$ into $\left(2 x, \frac{1}{2} y\right)$ if $0 \leqq x<\frac{1}{2}$ and $\left(2 x-1, \frac{1}{2} y+\frac{1}{2}\right)$ if $\frac{1}{2} \leqq x<1$. We can picture $T$ as follows: We first squeeze down the unit square and we then translate the part that has left the square back onto the part of the square that is now empty.

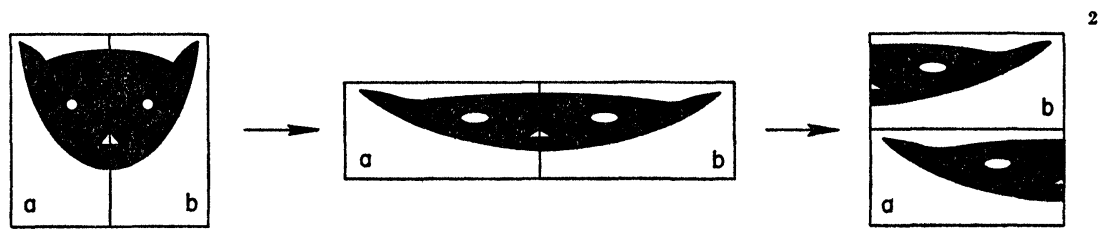

We describe the Bernoulli shift $\left(p_{1}, \cdots, p_{k}\right)$ in a similar way. $X$ is the unit square. Divide $X$ into $k$ rectangles whose height is 1 and whose base is an interval of length $p_{i}(1 \leqq i \leqq k)$. Squeeze the height of the $i$ th rectangle by $p_{i}$ and expand its width by $1 / p_{i}$. Now reassemble these pieces by putting the first on the bottom, the second on top of it, the third on top of that, etc.

There is a third way to describe Bernoulli shifts. $T$ is isomorphic to the Bernoulli shift $\left(p_{1}, \cdots, p_{k}\right)$ if and only if there is a partition $P$ into sets $P_{1}, \cdots, P_{k}$ such that the measure of $P_{i}$ is $p_{i}$ and such that:

(1) the $T^{i} P$ are independent and

(2) the $T^{i} P$ generate.

${ }^{2}$ This nice type of picture was suggested in [26]. 
(1) and (2) mean the following: (1) means that if we take a finite number of the $T^{i} P$ and pick one atom in each of the $T^{i} P$, then the measure of the intersection of these atoms will equal the product of their measures. (2) means that if $E$ is a measurable set, then, given $\epsilon$, we can find an $n$ and a set $E^{1}$ in the algebra of sets generated by the $T^{i} P,-n \leqq i \leqq n$, such that the symmetric difference between $E$ and $E^{\prime}$ is less than $\epsilon$. If $X$ is a Lebesgue space (isomorphic to the unit interval or the unit square with Lebesgue measure), then the above definition agrees with our previous definitions.

The isomorphism problem. When Halmos wrote his book on ergodic theory [1] one of the main problems was the following: Are all Bernoulli shifts isomorphic? In particular, is the Bernoulli shift $\left(\frac{1}{2}, \frac{1}{2}\right)$ isomorphic to the Bernoulli shift $\left(\frac{1}{3}, \frac{1}{3}, \frac{1}{3}\right)$ ? Up to this point there were various properties of transformations that were known, and because of these a large number of transformations could be distinguished from one another (properties such as ergodicity, mixing, weak mixing). None of these properties, however, distinguished any two Bernoulli shifts (two Bernoulli shifts even induced isomorphic unitary operators).

The breakthrough in this area came when Kolmogorov introduced [3] a new invariant called entropy, which was motivated by Shannon's work [4] on information theory. This invariant which I will describe below was easy to compute for the Bernoulli shift $\left(p_{1}, \cdots, p_{k}\right)$ and is simply $-\sum_{i=1}^{k} p_{i} \log p_{i}$. (Thus the Bernoulli shift $\left(\frac{1}{2}, \frac{1}{2}\right)$ is not isomorphic to the Bernoulli shift $\left(\frac{1}{3}, \frac{1}{3}, \frac{1}{3}\right)$.)

The entropy of a transformation is defined as follows: We first define the entropy, $H(P)$, of a partition $P$, whose $i$ th set has measure $p_{i}$ as $-\sum p_{i} \log p_{i}$. (If the $T^{i} P$ were independent, then for all $n$ large enough the size of most of the atoms in the partition $\bigvee_{i=1}^{n} T^{i} P$ is approximately $\frac{1}{2}^{H(P) n}$. This follows from the law of large numbers.) We now define the entropy of $P$ relative to $T, H(P, T)$ as $\lim \inf _{n \rightarrow \infty}(1 / n) H\left(\mathrm{~V}_{1}^{n} T^{i} P\right)$. Actually the limit exists. (There is a theorem due to Shannon and McMillan that says that for all $n$ large enough the size of most of the atoms in $\mathrm{V}_{i=1}^{n} T^{i} P$ is roughly the same and approximately equal to $\frac{1}{2} H\left(P, T^{T}\right)$.)

We now define $H(T)$ as sup $H(P, T)$ where sup is taken over all finite partitions. At first glance one would expect that $H(P, T)$ would be very large if $P$ had a large number of atoms and that $H(T)$ would always $=\infty$. To get a feeling why this is not so note that

$$
H(P, T)=\lim _{n \rightarrow \infty} \frac{1}{n} H\left(\bigvee_{1}^{n} T^{i} P\right)
$$


should equal

$$
H\left(\bigvee_{1}^{l} T^{i} P, T\right)=\lim _{n \rightarrow \infty} \frac{1}{n} H\left(\bigvee_{1}^{n+l} T^{i} P\right) .
$$

This same argument suggests that $\sup H(P, T)$ should be attained if the $T^{i} P$ generate. This is actually true (it would be very easy if each $B$ were actually in some $\mathrm{V}_{-n}^{n} T^{i} P$ ). It is this last fact that enables us to calculate the entropy for Bernoulli shifts.

Let us now return to the isomorphism problem for Bernoulli shifts. There are still quite a lot of Bernoulli shifts with a given entropy. Are these all the same? Is there another property yet to be discovered that will distinguish some of these? Are they all different? Mesalkin [6] showed that the answer to the last question was no by showing that the Bernoulli shifts $\left(\frac{1}{4}, \frac{1}{4}, \frac{1}{4}, \frac{1}{4}\right)$ and $\left(\frac{1}{2}, \frac{1}{8}, \frac{1}{8}, \frac{1}{8}, \frac{1}{8}\right)$ were isomorphic.

Sinai [5] proved a beautiful and deep theorem along these lines, namely: If $H(T)=-\sum p_{i} \log p_{i}$, then we can find a partition, $P$, whose $i$ th set has measure $p_{i}$ such that the $T^{i} P$ are independent. (The $T^{i} P$ may not generate. If this could be shown, under the additional hypothesis that $T$ is a Bernoulli shift, then all Bernoulli shifts of the same entropy would be isomorphic.)

We now know [7]

THEOREM. All Bernoulli shifts with the same entropy are isomorphic.

Various elaborations of the proof of the above theorem yield the following results.

Generalized Bernoulli shifts. In [7] we only consider the case where $P$ has a finite number of elements. Smorodinsky showed [8] that the argument in [7] could be modified to include the case where $P$ is countably infinite and $\sum-p_{i} \log p_{i}<\infty$. In [9] we show that any two Bernoulli shifts for which $\sum-p_{i} \log p_{i}=\infty$ are isomorphic. Actually, in [9] we prove a little more, and to state this result we will first define a generalized Bernoulli shift as follows. Let $S$ be a Lebesgue measure space of total measure 1 . Let $X$ be the product of a doubly infinite sequence of copies of $S$. For our measure on $X$ we will take the product measure. We define $T$, as before, to be the shift operator. Our result is that any two generalized Bernoulli shifts with the same (finite or infinite) entropy are isomorphic. [Note that if the measure on $S$ has a continuous part, then the entropy is infinite. Otherwise, $S$ has a countable number of points (or, after throwing away a set of measure $0, S$ has a countable number of points).]

Factors of Bernoulli shifts. In [10] we show that a factor of a Bernoulli shift is a Bernoulli shift. By this we mean the following: 
Let $T$ be a Bernoulli shift (or generalized Bernoulli shift). Let $a$ be a $\sigma$-algebra of measurable sets invariant under $T$. We can then find a partition $P$ (finite if the entropy of $T$ is finite, otherwise countable) such that the $T^{i} P$ are independent and generate $a$.

Bernoulli flows. Classifying the Bernoulli shifts gives information about them which at first glance one would not expect to get. For example, it was not previously known if the 2 -shift had a square root. We can now show it has a square root as follows. Let $T$ be a Bernoulli shift whose entropy is one-half that of the 2 -shift. It is easy to see that $T^{2}$ will be a Bernoulli shift with the same entropy as the 2 -shift. Therefore, the 2-shift has a square root. Similarly, Bernoulli shifts have roots of all orders and have lots of automorphisms that commute with them.

In [11] the above result is pushed further by showing that Bernoulli shifts can be imbedded in flows. The flow $S_{t}$ (which was previously shown by Totoki to be a $K$-automorphism for each $t$ [16]) can be described as follows: Let $T$ acting on $X$ be the 2-shift. Let $f$ be the function on $X$ that takes on two values: $\alpha$ on those points of $X$ whose first coordinate is 0 and $\beta$ on those points whose first coordinate is $1 . \alpha$ and $\beta$ are picked so that $\alpha / \beta$ is irrational. Let $Y$ be the area under the graph of $f . S_{t}$ will act on $Y$ as follows: each point, $(x, l)$, will move directly up at unit speed until it hits the graph of $f$. Then it goes to $(T x, 0)$ and continues moving up at unit speed. $S_{t}$ for each $t$ is shown to be a Bernoulli shift of finite entropy.

Smorodinsky and Feldman constructed a flow such that $S_{t}$ is a Bernoulli shift of infinite entropy.

We say $S_{t}$ is a Bernoulli flow if $S_{t}$ is a Bernoulli shift for some fixed $t$. In [30] we show that any two Bernoulli flows of finite entropy are isomorphic (by a trivial normalization we can assume $H\left(S_{1}\right)=1$ ).

Abstract ergodic theory : $K$-automorphisms. We will now consider the question: How large is the class of Bernoulli shifts or, equivalently, what is the class of transformations for which entropy tells the whole story? Kolmogorov had a very general conjecture along these lines which I would now like to describe. He introduced a class of transformations (now called $K$-automorphisms $-K$-transformations would be more consistent with the terminology of this article) and conjectured that if two $K$-automorphisms had the same entropy, they were isomorphic. (This would imply that they were Bernoulli shifts.)

We say that $T$ is a $K$-automorphism if there is a finite partition $P$ such that the $T^{i} P$ generate and $\bigcap_{n=1}^{\infty} \bigvee_{i=n}^{\infty} T^{i} P$ is trivial. (That is, $\mathrm{V}_{i=n}^{\infty} T^{i} P$ is the class of measurable sets generated by the $T^{i} P$, $n \leqq i<\infty$. The only sets which are contained in the above classes for 
all $n$ have either measure 0 or 1.) A special case is when the TiP generate and are independent.

There is a beautiful theorem due to Rohlin and Sinai [15] which says that $T$ is a $K$-automorphism if and only if for every finite partition $Q$ we have that $E(Q, T)>0$. (This is the same as saying that $Q$ is not contained in $\mathrm{V}_{-\infty}^{-1} T^{i} Q$.) This also implies that if $T$ is a $K$-automorphism, any finite partition satisfies the condition of the previous paragraph.

We now know that Kolmogorov's conjecture is false, [12], i.e.,

THEOREM. There is a K-automorphism that is not a Bernoulli shift.

The proof of the above theorem is closely related to the proof of the first theorem. We need a usable criterion for when the $T^{i} P$ generate a Bernoulli shift and such a criterion comes out of the methods used in the proof of the first theorem.

One of the main problems in ergodic theory is to find out what properties tell the whole story for $K$-automorphisms.

Pinsker's conjecture. Pinsker conjectured that every ergodic transformation of nonzero entropy is the direct product of a transformation of 0 -entropy and a $K$-automorphism. This would imply that the study of ergodic transformations reduced to the separate study of $K$-automorphisms and transformations of 0 -entropy. In [28] we give an example of a mixing transformation for which Pinsker's conjecture fails. This example is based on the example of a $K$-automorphism that is not a Bernoulli shift.

The root problem. In [1] Halmos raised the question, "Does every transformation with continuous spectrum have a square root and does every Bernoulli shift have a square root?" We already mentioned that the answer to the second question is yes. Chacon showed that the answer to the first question is no. In [29] we give an example of a $K$-automorphism with no square root. This shows roughly that no amount of mixing, unless $T$ is a Bernoulli shift, implies the existence of a square root. It also gives a qualitative way in which a $K$-automorphism can differ from a Bernoulli shift.

This example is a modification of the example of a $K$-automorphism that is not a Bernoulli shift.

Returning to the origins of the theory: random processes.

Codes. Now suppose $T$ is a Bernoulli shift and $P$ an arbitrary finite partition with $k$ atoms. I would like to describe the process $P, T$ in a somewhat more finitistic or physical way. For the sake of notation let us assume that $T$ is the Bernoulli shift $\left(\frac{1}{2}, \frac{1}{2}\right)$. Thus $T$ is the shift based 
on flipping a coin. To each sequence of 0,1 's we will associate another sequence as follows: Fix $n$ and $k$ and a definite way of dividing all possible sequences of 0,1 's of length $2 n+1$ into $k$ classes $1, \cdots, k$. For each term in our infinite sequence of 0,1 's look at the block of $2 n+1$ consecutive terms with it as center and print out the corresponding $1, \cdots, k$. Call the resulting mapping (of sequences of 0,1 's to sequences of $1, \cdots, k$ 's) a "code" of length $n$. If we code the output of our process we get another process. (The coding is physically realizable if we allow a time delay.) We say that a sequence of longer and longer codes (of the Bernoulli shift $\left(\frac{1}{2}, \frac{1}{2}\right)$ ) converges if, for each fixed term, all sufficiently long codes give the same result (except for terms belonging to a collection of sequences of 0,1 's of probability 0 ). This implies that any two sufficiently long codes agree with very high probability. It is easy to see that if $T$ is the Bernoulli shift $\left(\frac{1}{2}, \frac{1}{2}\right), B$ its independent generator, and $P \subset V_{-k}^{k} T^{i} B$ for some $k$, then $P, T$ can be realized by coding $B, T$. Thus $P, T$ can be physically constructed from a roulette wheel. It is easy to see that any process $P, T$ ( $T$ the Bernoulli shift $\left(\frac{1}{2}, \frac{1}{2}\right)$ ) is obtained either by coding the sequence of 0,1 's or as the limit of a convergent sequence of longer and longer codes. It is also easy to see that, in general, if two processes generate isomorphic transformations, then we can obtain one from the other by means of a convergent sequence of codes (and then get back the original process exactly by applying another convergent sequence of codes). Thus the isomorphism theorem for Bernoulli shifts can be interpreted in terms of codes.

Can any completely nondeterministic process be constructed from a roulette wheel? If the process $P, T$ has 0 -entropy $(H(P, T)=0)$, then $P, T$ is deterministic in the following sense: $P \subset \mathrm{V}_{-\infty}^{-1} T^{i} P$, which means that by knowing the past we can predict the next letter with probability 1 , or by knowing enough of the past we can predict the next letter with arbitrarily high probability. We can even make a stronger statement. For all $n, P \subset V_{-\infty}^{-n} T^{i} P$, which means that we can predict a particular letter by knowing the distant past, however distant.

Now suppose $T$ is a $K$-automorphism and $P$ any finite partition, such that the $T^{i} P$ generates. Let us call such a process a $K$-process. The $K$-processes are exactly those processes that contain no deterministic part or are completely nondeterministic in the following sense: If $P, T$ is not a $K$-process (and $T$ is ergodic ${ }^{3}$ ), then for some $N$ we can divide the sequences of length $N$ into two classes (each

${ }^{3}$ The assumption is there only for simplicity. In fact, if $T$ is not ergodic, we have an even stronger deterministic property. 
having measure between $1 / 2-1 / 100$ and $1 / 2+1 / 100$ ) and we predict with probability $99 / 100$ which class the next $N$ symbols will belong to by knowing the distant past, however distant. (All we really need to know is a finite number of terms in the distant past; however, we need more terms in the more distant past.)

In these terms Kolmogorov's conjecture says that if $P, T$ is completely nondeterministic, then $T$ is a Bernoulli shift and the process is essentially constructible from a roulette wheel. The fact that Kolmogorov's conjecture is false means that we have

Theorem. There exists a completely nondeterministic process, $\bar{P}, \bar{T}$, such that if $T$ is a Bernoulli shift (and $P$ a finite partition), then for all $n$ large enough we can find two disjoint collections of sequences of length $n$, $C$ and $\bar{C}$, such that the measure of $\bar{C}$ under $\bar{P}, \bar{T}$ is greater than $99 / 100$ and the measure of $C$ under $P, T$, is greater than 99/100.

(It turns out that even more is true. There is a fixed $\sigma>0$, associated with $\bar{P}, \bar{T}$, such that $C$ and $\bar{C}$ can be chosen with the additional property that any sequence in $C$ differs from any sequence in $\bar{C}$ in more than $\sigma n$ places. Thus $\bar{P}, \bar{T}$ and $P, T$ are a definite distance apart (see [14]).)

The above theorem says that there is a completely nondeterministic process that is a definite distance away from all processes that can be constructed from roulette wheels.

In terms of the above notion of distance it can be shown [14] that the processes $(P, T), T$ a Bernoulli shift, $P$ an arbitrary partition, are the only ones that can be approximated arbitrarily well by processes physically constructible from a roulette wheel.

Pinsker's conjecture. If this were true, then any process could be constructed from a deterministic and a completely nondeterministic process.

Markov processes. We say that a process is a Markov process if the probability of printing a given letter depends on the previous letter but not on any of the ones before that. It was shown (in a joint paper with $\mathrm{N}$. A. Friedman [13]) that if $P, T$ is a mixing ${ }^{4}$ Markov process and $P$ generates, then $T$ is a Bernoulli shift.

The criterion developed in the above mentioned paper seems to apply to the situation where $P, T$ is the model for the process obtained by observing a teleprinter, implying that $T$ is a Bernoulli shift. Since the above is clearly not a precise mathematical statement all that can be said is that it seems reasonable.

McCabe-Shields [17] and Smorodinsky [18] have extended the

- Mixing in this context is equivalent to saying that if $N$ is large enough, then any letter is possible at time $N$, regardless of what letter was printed out at time 0 . 
Markov process result to certain Markov processes with continuous state space. The class contains the regular Gaussian processes.

Returning to the origins of the theory : automorphisms of compact groups. Let $R$ be the transformation defined on the unit square as follows: $R(x, y)=(x+y, x+2 y)(\bmod 1)$. It is easy to see that $R$ is an invertible, measure-preserving transformation. The criterion developed in [11], [13] shows that $R$ is (i.e., isomorphic to, see p. 878) a Bernoulli shift.

$R$ is an example of the following more general situation. Take any $2 \times 2$ matrix with integer coefficients and determinants \pm 1 . (In the case of $R$ we take $\left(\begin{array}{ll}1 & 1 \\ 1 & 2\end{array}\right)$.) Such a matrix acts on the unit square by transforming each point and then subtracting off the largest integer in the $x$-coordinate and the largest integer in the $y$-coordinate. Because the determinant is \pm 1 , this transformation is measure-preserving and invertible. It can be shown [1] that the transformation is ergodic if and only if the matrix has no eigenvalues that are roots of unity. In this case our criterion applies and we can show that we get a Bernoulli shift.

Let us note that the unit square is a topological group under addition mod 1 (usually called the 2-dimensional torus) and that the transformations described above are its automorphisms. We thus have that the ergodic automorphisms of the 2-dimensional torus are Bernoulli shifts. (Adler and Weiss already showed that entropy classified the automorphisms of the 2-dimensional torus [19].)

Using results of Sinai [20] it could be shown that an automorphism of the $n$-dimensional torus whose matrix had no eigenvalues on the unit circle was a Bernoulli shift. The case of eigenvalues on the unit circle that were not roots of unity (the automorphism is ergodic if and only if there are no eigenvalues that are roots of unity) was still a mystery. We now have

ThEOREM (KATZNELSON [21]). Every ergodic automorphism of the n-dimensional torus is isomorphic to a Bernoulli shift.

Since the entropy can be calculated in terms of the eigenvalues of the matrix (see Sinai [20] and Bowen [22]), Katznelson's theorem tells us exactly what the automorphism is, measure-theoretically.

As far as general compact groups go, Rohlin [23] and Juzvinskii [24] showed years ago that every ergodic automorphism of a compact group is a $K$-automorphism. It is still not known if they are Bernoulli shifts.

Returning to the origin of the theory: mechanical systems. So far there is only one kind of mechanical system that can be completely 
analyzed from the ergodic theory point of view (that is, we know exactly-up to isomorphism-what the transformation on phase space is). These systems are the so-called geodesic flow on surfaces of negative curvature. I call these mechanical systems because ${ }^{5}$ in 3-dimensional Euclidean space one can specify a closed surface $V$ and place near it a finite number of centers of attraction or repulsion, creating a force potential in $V$ such that the motion of a material point constrained to move in $V$ under the above potential will be mathematically equivalent to geodesic flow on a surface of negative curvature.

By putting together results of Sinai and Anosov (see Arnold and Avez [26]) and the criterion that comes from the proof of the isomorphism theorem for Bernoulli shifts we get that the above flows are Bernoulli flows (restricted to a surface of constant energy).

What do the above results mean? Suppose that we perform an experiment or take some measurement of our mechanical system and that this experiment or measurement has only a finite number of possible outcomes. Suppose also that we repeat this measurement at regularly spaced intervals of time (all multiples of some fixed unit of time). The sequence of outcomes gives us a process. In some sense all the information we can get about a mechanical system is some process derived from it. Our result says that these processes are very random and cannot be distinguished from processes obtained by coding a roulette wheel.

Geodesic flow on manifolds of negative curvature are very special examples. There is, however, reason to believe that they are just mathematically simpler cases of a fairly general phenomenon, which includes the case of hard sphere gas enclosed in a box. I should mention in this connection that Sinai has succeeded in proving that the hard sphere gas in a rectangular box is ergodic on manifolds of constant energy. He actually proved more, namely that the above transformations are $K$-automorphisms [27].

\section{REFERENCES}

1. P. R. Halmos, Lectures on ergodic theory, Publ. Math. Soc. Japan, no. 3, Math. Soc. Japan, Tokyo, 1956; reprint, Chelsea, New York, 1960, pp. 54-55. MR 20 \#3958.

2. P. Billingsley, Ergodic theory and information, Wiley, New York, 1965. MR 33 \#254.

3. A. N. Kolmogorov, $A$ new metric invariant of transient dynamical systems and automorphisms in Lebesgue spaces, Dokl. Akad. Nauk SSSR 119 (1958), 861-864. (Russian) MR 21 \#2035a.

5 This was pointed out by Kolmogorov in [25]. 
4. - Entropy per unit time as a metric invariant of automorphisms, Dokl. Akad. Nauk SSSR 124 (1959), 754-755. (Russian) MR 21 \#2035b; 21, 1599.

5. Ja. G. Sinar, $A$ weak isomorphism of transformations with an invariant measure, Dokl. Akad. Nauk SSSR 147 (1962), 797-800=Soviet Math. Dokl. 3 (1962), 17251729. MR 28 \#5164a; 28 \#1247.

6. L. D. Mešalkin, A case of isomorphism of Bernoulli schemes, Dokl. Akad. Nauk SSSR 128 (1959), 41-44. (Russian) MR 22 \#1650.

7. D. S. Ornstein, Bernoulli shifts with the same entropy are isomorphic, Advances in Math. 4 (1970), 337-352. MR 41 \#1973.

8. M. Smorodinsky, An exposition of Ornstein's isomorphism theorem, Advances in Math. (to appear).

9. D. S. Ornstein, Two Bernoulli shifts with infinite entropy are isomorphic, Advances in Math. 5 (1970), 339-348.

10. - Factors of Bernoulli shifts are Bernoulli shifts, Advances in Math. 5 (1970), 349-364.

11. - Imbedding Bernoulli shifts in flows, Contributions to Ergodic Theory and Probability, Lecture Notes in Math., Springer-Verlag, Berlin, 1970, pp. 178-218.

12. - A simpler example of a Kolmogorov automorphism that is not a Bernoulli shift, Advances in Math. (to appear).

13. N. A. Friedman and D. S. Ornstein, On isomorphism of weak Bernoulli transformations, Advances in Math. 5 (1970), 365-394.

14. D. S. Ornstein, An application of erogdic theory to probability theory, Advances in Math. (to appear).

15. V. A. Rohlin and Ja. G. Sinal, Construction and properties of invariant measurable partitions, Dokl. Akad. Nauk SSSR 141 (1961), 1038-1041 = Soviet Math. Dokl. 2 (1961), 1611-1614. MR 27 \#2604.

16. S. Ito, H. Murata and $\mathrm{H}$. Totoki, A remark on the isomorphism theorem for weak Bernoulli transformations (to appear).

17. R. McCabe and P. Shields, A class of Markov shifts which are Bernoulli shifts, Advances in Math. (to appear).

18. M. Smorodinsky, A partition for a Bernoulli shift that is not weak Bernoulli (to appear).

19. R. L. Adler and B. Weiss, Entropy, a complete metric invariant for automorphisms of the torus, Proc. Nat. Acad. Sci. U.S.A. 57 (1967), 1573-1576. MR 35 \#3031.

20. Ja. G. SinaĬ, Markov partitions and Y-diffeomorphisms, Funkcional. Anal. i Priložen. 2 (1968), no. 1, 64-89=Functional Anal. Appl. 2 (1968), 61-82. MR 38 \#1361.

21. Y. Katznelson, Ergodic automorphisms of $T^{n}$ are Bernoulli shifts, Israel J. Math. (to appear).

22. R. Bowen, Entropy for group endomorphisms and homogeneous spaces, Trans. Amer. Math. Soc. 153 (1971), 401-414.

23. V. A. Rohlin, Metric properties of endomorphisms of compact commutative groups, Izv. Akad. Nauk SSSR Ser. Mat. 28 (1964), 867-874; English transl., Amer. Math. Soc. Transl. (2) 64 (1967), 244-252. MR 29 \#5955.

24. S. A. Juzvinskil, Metric properties of endomorphisms of compact groups, Izv. Akad. Nauk SSSR Ser. Mat. 29 (1965), 1295-1328; English transl., Amer. Math. Soc. Transl. (2) 66 (1968), 63-98. MR 33 \#2798.

25. A. N. Kolmogorov, Théorie générale des systèms dynamiques et mécanique classique, Proc. Internat. Congress Math. (Amsterdam), vol. 1, North-Holland, Amsterdam, 1957, pp. 315-333. MR $20 \# 4066$. 
26. V. I. Arnold and A. Avez, Ergodic problems of classical mechanics, Benjamin, New York, 1968. MR 38 \#1233.

27. Ja. G. Sinal, On the foundations of the ergodic hypothesis for a dynamical system of statistical mechanics, Dokl. Akad. Nauk SSSR 153 (1963), 1261-1264=Soviet Math. Dokl. 4 (1963), 1818-1822. MR 35 \#5576.

28. D. S. Ornstein, A mixing transformation for which Pinsker's conjecture fails (to appear).

29. — , A K-automorphism with no square root and Pinsker's conjecture (to appear).

30. - The isomorphism theorem for Bernoulli flows (to appear).

Stanford University, Stanford, California 94305 